\title{
Quality of life after gamma knife radiosurgery treatment in patients with a vestibular schwannoma: the patient's perspective
}

\author{
Ferdinand C. A. Timmer • Anniek E. P. van Haren · Jef J. S. Mulder • Patrick E. J. Hanssens • \\ Jacobus J. van Overbeeke $\cdot$ Cor W. R. J. Cremers $\cdot$ Kees Graamans
}

Received: 20 July 2009/Accepted: 16 October 2009/Published online: 6 November 2009

(C) The Author(s) 2009. This article is published with open access at Springerlink.com

\begin{abstract}
This study evaluates the impact of gamma knife radiosurgery (GKRS) on the quality of life (QOL) of patients with a sporadic vestibular schwannoma (VS). This study pertains to 108 VS patients who had GKRS in the years 2003 through 2007. Two different QOL questionnaires were used: medical outcome study short form 36 (SF36) and Glasgow benefit inventory (GBI). Radiosurgery was performed using a Leksell $4 \mathrm{C}$ gamma knife. The results of the QOL questionnaires in relation to prospectively and retrospectively gathered data of the VS patients treated by GKRS. Eventually, 97 patients could be included in the study. Their mean tumor size was $17 \mathrm{~mm}$ (range 6-39 $\mathrm{mm}$ ); the mean maximum dose on the tumor was 19.9 Gy (range 16-25.5 Gy) and the mean marginal dose on the tumor was 11.1 (range 9.3-12.5 Gy). SF36 scores showed results comparable to those for a normal Dutch population. GBI showed a marginal decline in QOL. No correlation was found between QOL and gender, age, tumor size, or radiation dose. Increased audiovestibular
\end{abstract}

F. C. A. Timmer $(\bowtie)$ · A. E. P. van Haren .

J. J. S. Mulder - C. W. R. J. Cremers - K. Graamans

Department of Otorhinolaryngology, Head and Neck Surgery,

Donders Institute for Brain, Cognition and Behavior,

Radboud University Nijmegen Medical Center,

PO BOX 9101, 6500 HB Nijmegen, The Netherlands

e-mail: f.timmer@kno.umcn.nl

P. E. J. Hanssens

Gamma Knife Center Tilburg, St Elisabeth Hospital,

Tilburg, The Netherlands

J. J. van Overbeeke

Department of Neurosurgery, Gamma Knife Center Tilburg,

St Elisabeth Hospital, Tilburg, The Netherlands symptoms after GKRS were correlated with a decreased GBI score, and decreased symptoms were correlated with a higher QOL post-GKRS. In this study shows that GKRS for VS has little impact on the general QOL of the VS patient. However, there is a wide range in individual QOL results. Individual QOL was influenced by the audiovestibular symptoms. No predictive patient, tumor, or treatment factors for QOL outcome after GKRS could be determined. Comparison with microsurgery is difficult because of intra group variability.

Keywords Vestibular schwannoma - Acoustic neuroma . Gamma knife · Radiosurgery - Complications .

Quality of life

\section{Introduction}

A vestibular schwannoma (VS) is a benign tumor arising from the vestibular part of the eighth cranial nerve. Unilateral hearing loss, tinnitus, and unsteadiness are the most common symptoms at the time of diagnosis [1]. These symptoms influence the quality of life (QOL) of the VS patient, as has been described in earlier papers $[2,3]$. Tumor size is not found to be related to the amount and severity of audiovestibular symptoms [3]. The growth pattern of VSs is unpredictable. A large proportion of all VSs show no growth in the first years after diagnosis, and a regression of tumor size has even been described in some cases. When diagnosed, VS is rarely a life-threatening condition, but large VSs need treatment to prevent brainstem compression. Due to the increased availability of magnetic resonance imaging (MRI), VSs can now be diagnosed at an earlier stage and their growth can be monitored at regular intervals. 
The treatment strategy after the initial diagnosis is aimed at controlling tumor growth and preserving cranial nerve functions. Treatment options include conservative management (wait and scan, W\&S), microsurgery (MS), and stereotactic radiation therapy. A well-known type of stereotactic radiation therapy is gamma knife radiosurgery (GKRS).

It has become a prominent treatment option for $\mathrm{VS}<3 \mathrm{~cm}$. Its tumor control rate is assumed to be comparable to that achieved by microsurgery, but the impact on QOL appears to be less [4, 5]. Until the end of the twentieth century, the minimal (12-20 Gy) and maximal (24-50 Gy) tumor doses were relatively high and the planning was less precise by current standards [6]. Whereas side effects and complications related to GKRS seemed to be significant in the past, nowadays, the GKRS seems to be a treatment option that does not pose any serious risks.

This study was carried out to evaluate the impact of GKRS on the QOL of VS patients. The aim was to evaluate audiovestibular symptoms and to correlate this data with QOL parameters.

\section{Patients and methods}

Study population and study design

This study pertains to 108 patients who had GKRS in the years 2003 through 2007. They were initially seen and examined at the Department of Otorhinolaryngology, Head and Neck Surgery of our tertiary referral center. Among the reasons to proceed to GKRS were MRI-proven tumor growth $(>2 \mathrm{~mm}$ difference in maximal diameter of the tumor in either direction on sequential MR images in the axial plane) and the patient's personal preference after consultation.

Together with an information letter and questionnaire about audiovestibular symptoms before and after treatment with GKRS, two different QOL questionnaires were sent to the patients after GKRS. Those unable to answer the questionnaires were excluded from the study.

Quality of life questionnaires

Two questionnaires were used: the medical outcome study short form 36 (SF36) and the Glasgow benefit inventory (GBI). The first questionnaire, the SF36, was originally constructed to survey health status in the Medical Outcomes Study [7]. The short form 36 health survey comprises 36 items, non-specific for disease, with two to six response choices per item. The pre-coded responses are recoded in percentages. Items falling into the same category are averaged to create eight health concepts: physical functioning, bodily pain, role limitations due to physical health problems, role limitations due to personal or emotional problems, emotional well-being, social functioning, vitality, and general health perceptions. The scores are presented with a minimum of zero and a maximum of 100. A higher score means a higher health state. Dutch norm scores are available for reference purposes [8].

The second questionnaire is the Glasgow benefit inventory (GBI), a tool to measure patient benefit that was developed especially for otorhinolaryngological interventions [9]. The questionnaire contains 18 post-intervention items, eliciting responses based on a five-point Likert scale. This scale ranges from a low health status, expressed in a low score, to a high health status. In this case, the items pertain to GKRS. Various scores can be calculated from the item responses: the total score, general subscale score, social support subscale score, and physical health subscale score. The scores range from -100 to 100 , with a score of 0 indicating no health change after an intervention and -100 , a worsened health state after treatment.

\section{Tumor size}

Tumor size was determined according to the consensus reached in Tokyo on 7 November 2001 [10]. In the case of extrameatal tumors, this involved measuring the largest extrameatal diameter on axial MR images. Intrameatal tumors were measured parallel to the internal acoustic meatus (IAM). The intra- and extrameatal portions of the tumor were clearly delineated by the continuation of the line of the petrous ridge depicted on serial axial MR images. A tumor was classified as an intrameatal lesion when there was no tumor extension beyond that plane. All other tumors were recorded as extrameatal lesions.

Complications and audiovestibular symptoms

There are several possible complications of GKRS: sensorineural impairment, vestibular disorders, facial nerve paresis (including lacrimal gland dysfunction), trigeminal nerve dysfunction (disturbances of facial sensibility and facial pain), recurrent tumor growth, headache, and complications due to installing the stereotactic frame on the head.

Since most of these complications could also be caused by the VS itself, the symptoms before GKRS were compared to those after GKRS. Patients were retrospectively asked to indicate an increase, decrease, or no change of symptoms. Furthermore, all patient charts were checked for other complications or side effects of GKRS. 
Gamma knife settings, procedure, and cochlear radiation dose

A Leksell titanium stereotactic frame was installed on each patient's head after injecting a local anesthetic subcutaneously at the screw points in the skull. The patient was then placed in a Philips T1.0 MRI scanner. Axial T1 $1.0 \mathrm{~mm}$ MR images were made before and after administering gadolinium contrast. Then 3D TSE (Turbo Spin Echo) $0.7 \mathrm{~mm}$ images were constructed.

Using Leksell gamma-plan software V5.34, the tumor was delineated on the corresponding images using the TSE and T1-weighted gadolinium contrast enhanced images. The tumor volume was then calculated using the 3D software. A radiation plan was made by placing 'isocenters' or 'shots' in the tumor volume, guided by prescribed dose (PD), marginal dose, and conformity. The cochlea was pointed out on each axial MR image, and the maximal radiation dose on the cochlea was calculated.

Stereotactic surgery was performed using a Leksell 4C gamma knife. Treatment time depended on the radioactivity of the sources and the number of shots. That number was determined on the grounds of the tumor's volume and configuration. Patients were discharged from the hospital the same day.

\section{Statistics}

Statistical analysis was performed with SPSS (16.0). Statistical significance was set at the 5\% level. Summary statistics (mean, SD, range) were expressed as a frequency distribution. As the results of the questionnaires were skewed, non-parametric tests were carried out to describe the difference between groups. The Mann-Whitney $U$ test was used to compare two groups, the Kruskal-Wallis test to compare more than two. Spearman's rho was used for continuous data. Even if the patients failed to complete the entire questionnaire, the available data was used in the calculations.

\section{Results and analysis}

\section{Group characteristics}

Of the initial 108 patients who underwent GKRS between 2003 and 2007, one patient had to be excluded since he was unable to answer the questions due to dementia, presumably not related to the VS or GKRS. Questionnaires were returned by 97 of the remaining 107 patients $(91 \%$ response rate).

The responders had received the questionnaires after an average period of 21 months (range 2-55 months) following GKRS. The study group's characteristics are outlined in Table 1. The gamma knife settings and tumor radiation doses are given in Table 2 .

\section{General quality of life after GKRS}

The general QOL after GKRS of our study group was assessed with the SF36 questionnaire. Here, the results are compared to those for a normal Dutch population [8]. A $t$ test showed that the RP and GH domains were significantly lower in our study group compared to the normal population. The population characteristics and SF36 results for the normal population and the study population are given in Table 3.

\section{Quality of life change after treatment}

The GBI compared retrospectively the QOL of patients before and after treatment with GKRS. Table 4 outlines the results of the study population for this questionnaire. The total scores and the three subscale scores are shown.

The mean total GBI score of -0.1 indicates a slight negative change in the overall QOL after GKRS. The mean social support score was above zero, which indicates better social support after treatment. There was no significant difference in mean total GBI score between patients who received the questionnaires within 12 months after GKRS treatment and those who received the questionnaires later $(P=0.409$, Mann-Whitney $U$ test $)$.

No significant difference was found between men and woman for the mean total GBI score $(P=0.516$, MannWhitney $U$ test). The GBI scores for patients younger than 40 years, patients from 40 to 60 , and patients over 60 are shown in Fig. 1. There seems to be a tendency toward better QOL in older patients. However, significance was not reached in any of the domains.

The GBI scores calculated for patients with tumors smaller than $10 \mathrm{~mm}$, between 10 and $20 \mathrm{~mm}$, and larger

Table 1 Descriptive statistics of the study group

\begin{tabular}{lc}
\hline Sex & 52 Male/45 female \\
Mean age at VS diagnosis (years) & 53 (range 23-81) \\
Mean age at GKRS (years) & 56 (range 24-84) \\
Mean time between diagnosis and & 27 (range 2-126) \\
$\quad$ GKRS (months) & 58 Right $(59.8 \%)$, \\
Tumor side & 39 left $(40.2 \%)$ \\
& 94 Extrameatal $(96.9 \%)$, \\
Tumor location & 3 intrameatal $(3.1 \%)$ \\
& 17 (range 6-39) \\
$\begin{array}{l}\text { Mean tumor size extrameatal (mm) } \\
\text { Mean tumor volume before GKRS } \\
\left(\mathrm{mm}^{3}\right)\end{array}$ & 2,721 (range 25-17,700) \\
\hline
\end{tabular}


Table 2 Gamma knife settings and dosages at the tumor

\begin{tabular}{llcl}
\hline & Mean (Gy) & Range (Gy) & SD \\
\hline Marginal dose & 11.1 & $9.3-12.5$ & 0.45 \\
Tumor dose 90\% & 12.7 & $12-13.2$ & 0.33 \\
Maximal tumor dose & 19.9 & $16-25.5$ & 1.7 \\
Maximal cochlea dose & 10.4 & $5-16.1$ & 2.69
\end{tabular}

The marginal dose is defined as the minimal dose received by $100 \%$ of the tumor. Tumor dose $90 \%$ is the amount of Gy minimally received by $90 \%$ of the tumor

than $20 \mathrm{~mm}$ did not show a significant correlation between the mean total score $(P=0.57$ Kruskal-Wallis test $)$ and other scores. Figure 2 shows the different GBI scores for patients classified by tumor size.

No correlation was found between the mean total GBI score for maximal cochlea $(P=0.060$, Spearman's rho) or maximal tumor radiation dose $(P=0.365$, Spearman's rho). In addition, no significance was reached for the mean total GBI score and initial management; W\&S or immediate GKRS after diagnosis $(P=0.201$, Mann-Whitney $U$ test).

The outcome of the audiovestibular symptom questionnaire is presented in Table 5. In retrospect, the patients had their symptoms both before and after GKRS. Patients who did not have a specific symptom before treatment but did have it after treatment were placed in the 'increased' group.

Table 3 SF36 study population and Dutch normal population

\begin{tabular}{|c|c|c|c|}
\hline & $\begin{array}{l}\text { Study } \\
\text { population } \\
n=97\end{array}$ & $\begin{array}{l}\text { Normal } \\
\text { population }[8] \\
n=1,742\end{array}$ & $\begin{array}{l}\text { Unpaired } \\
t \text { test }(P \text { value })\end{array}$ \\
\hline \multicolumn{4}{|c|}{ Age receiving questionnaire } \\
\hline Mean (SD) & $57.6(13.3)$ & $47.6(18.0)$ & $<0.0001$ \\
\hline Range & $26-86$ & $16-94$ & \\
\hline \multicolumn{4}{|l|}{ Sex } \\
\hline Male & $54 \%$ & $56 \%$ & \\
\hline Female & $46 \%$ & $44 \%$ & \\
\hline \multicolumn{4}{|l|}{ SF36 } \\
\hline PF mean (SD) & $82.5(20.9)$ & $83.0(22.8)$ & 0.83 \\
\hline RP mean (SD) & $68(40.6)$ & $76.4(36.3)$ & $\mathbf{0 . 0 3}$ \\
\hline RE mean (SD) & $80.7(36.2)$ & $82.3(32.9)$ & 0.64 \\
\hline VT mean (SD) & $66.3(22.4)$ & $68.6(19.3)$ & 0.25 \\
\hline MH mean (SD) & $80.1(15.8)$ & $76.8(17.4)$ & 0.06 \\
\hline SF mean $(\mathrm{SD})$ & $81.3(22.1)$ & $84.0(22.4)$ & 0.24 \\
\hline BP mean (SD) & $78.4(22.1)$ & $74.9(23.4)$ & 0.15 \\
\hline GH mean $(\mathrm{SD})$ & $65.5(20)$ & 70.7 (20.7) & 0.01 \\
\hline
\end{tabular}

$P F$ physical functioning, $R P$ role limitations physical, $R E$ role limitations emotional, $V T$ vitality, $M H$ general mental health, $S F$ social functioning, $B P$ bodily pain, $G H$ general health, $P H$ physical health, $E H$ emotional health, $S D$ standard deviation

Significant values are given in bold
Table 4 GBI total and subscale scores

\begin{tabular}{llll}
\hline & Mean $(n)$ & SD & Range \\
\hline Study population $(n=97)$ & & & \\
$\quad$ Total score & $-0.1(71)$ & 14.6 & -33.3 to 52.8 \\
General subscale score & $-1.4(78)$ & 19.3 & -50 to 62.5 \\
Social support subscale score & $+6.7(94)$ & 18.8 & -33.3 to 100 \\
Physical health subscale score & $-4.9(91)$ & 12.5 & -50 to 33.3 \\
\hline
\end{tabular}

Based on the audiovestibular questionnaire, the study group was divided into three subgroups. Figure 3 shows the GBI scores of the patients with increased, decreased, or unaltered severity of the most common audiovestibular symptoms in the population: tinnitus, hearing impairment, and unsteadiness/vertigo. The mean general and total GBI scores were significantly higher when there was a total decrease in the severity of the three symptoms. In case of an increase in severity, the social support score was higher and the physical score was lower, although this was not significant for both findings.

Compared with the maximal dosages at the tumor and the cochlea, there is no positive relation with the groups reporting decreased, unaltered, or increased symptoms. A higher dose was not related to an increase in symptoms as assessed with the audiovestibular symptom questionnaire. No secondary interventions because of recurrent tumor growth have occurred so far.

\section{Discussion}

In our study population, we found that GKRS has a small impact on the general QOL in VS patients. The QOL of VS patients after GKRS was comparable to that of a normal

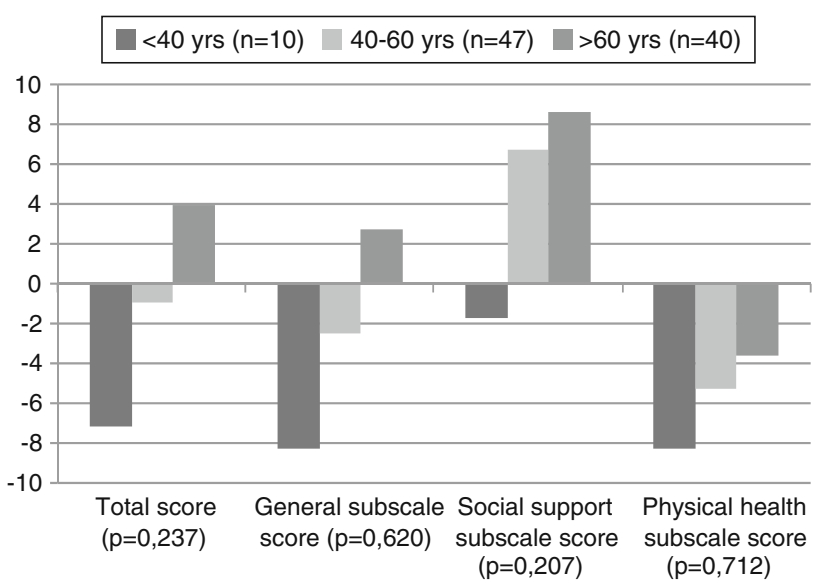

Fig. 1 GBI total and subscale scores for patients younger than 40 years, patients between 40 and 60 years, and patients older than 60 years. Significance assessed by the Kruskal-Wallis test 


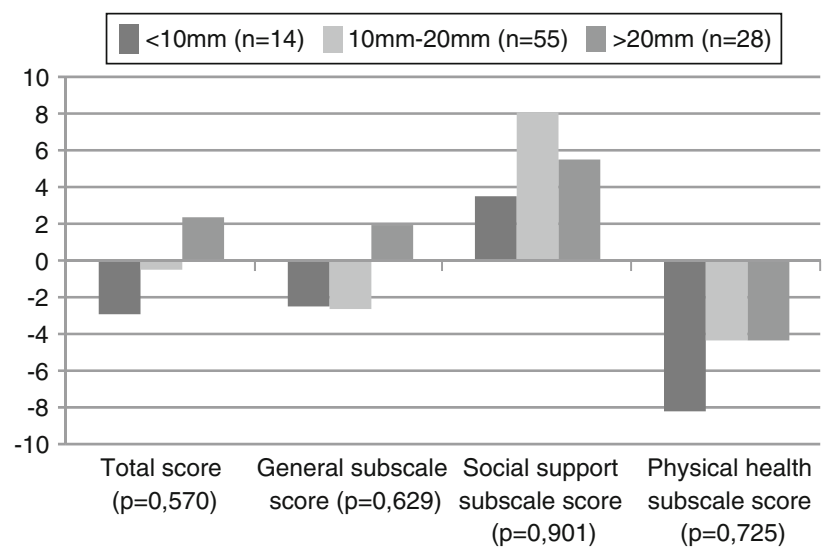

Fig. 2 GBI total and subscale scores for patients with tumors smaller than $10,10-20 \mathrm{~mm}$, and larger than $20 \mathrm{~mm}$. Significance tested by the Kruskal-Wallis test

Dutch population, as measured with the SF36 questionnaire. Only the RP and GH domains were significantly lower in our study group compared to the normal population.

According to the mean total GBI score, the impact of GKRS on the QOL was negligible. However, there is a wide range in individual QOL results and a clear correlation was found between the change in severity of audiovestibular complaints and the total GBI score. Our measurements demonstrated that hearing symptoms were unaltered in about $72 \%$, increased in $22 \%$, and decreased in $7 \%$ of the VS patients after GKRS.

Measuring QOL and comparing QOL study outcomes is difficult. The timing of the interview and the questions asked may be decisive for the patient's responses. Tumor size often differs between study groups. The post-treatment QOL outcome could be influenced by the doctor's social and surgical skills as well as by recurrent tumor growth after GKRS or MS. In general, each selection bias or comorbidity difference between different groups makes comparison of these groups questionable [11]. Despite these drawbacks, we think it is useful to take note of some other studies, which used the same questionnaires, as they put our findings in perspective.

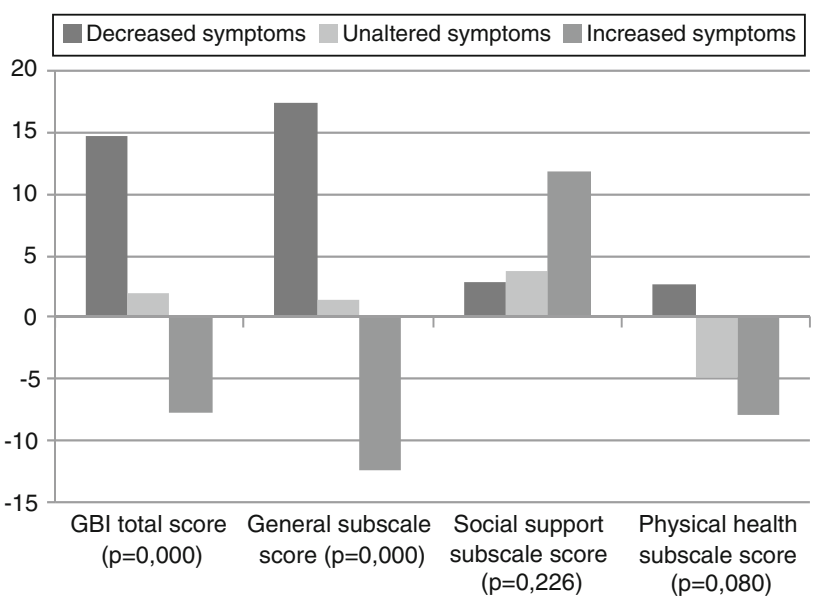

Fig. 3 Mean GBI scores for total decrease, increase, or no change in tinnitus, hearing, and unsteadiness. ${ }^{* *} P<0.01$ (Kruskal-Wallis test)

\section{SF36}

The SF36 results measured in our study group are given in Table 6 together with values for the normal Dutch population. These results are placed alongside the SF36 results found by other authors after MS, and after MS with VS larger than $20 \mathrm{~mm}$ extrameatal diameter.

Our scores on the SF36 questionnaire after GKRS were very similar to those for a normal Dutch population. This suggests the presence of a small impact on the general QOL for VS patients who underwent GKRS and therefore also for VS patients in general.

In the other two studies after MS in VS patients, lower scores were found in all domains compared to our postGKRS group [2, 12]. Conclusions based on comparisons between these groups and our study group are questionable because of differences in tumor size and follow-up time. Myrseth et al. [13] recently published a prospective nonrandomized study. They also used the SF-36 to compare QOL between GKRS and MS and did not find any trend toward a better or worse outcome for either treatment group after 2 years of follow-up.
Table 5 Audiovestibular symptoms before and after GKRS, and change in symptoms after treatment

\begin{tabular}{lllllc}
\hline$n=97$ & $\begin{array}{l}\text { Number of } \\
\text { patients } \\
\text { Before GKRS }(\%)\end{array}$ & $\begin{array}{l}\text { Number of } \\
\text { patients } \\
\text { After GKRS }(\%)\end{array}$ & $\begin{array}{l}\text { Decreased } \\
\text { symptom } \\
(\%)\end{array}$ & $\begin{array}{l}\text { Unaltered } \\
(\%)\end{array}$ & $\begin{array}{l}\text { Increased } \\
\text { symptom (\%) }\end{array}$ \\
\hline Tinnitus & $78(80)$ & $84(87)$ & $6(6)$ & $67(69)$ & $24(25)$ \\
Hearing impairment & $89(92)$ & $93(96)$ & $6(6)$ & $70(72)$ & $21(22)$ \\
Unsteadiness/vertigo & $64(66)$ & $73(75)$ & $7(7)$ & $69(71)$ & $21(22)$ \\
Facial function & $3(3.1)$ & $10(10)$ & 0 & $89(92)$ & $8(8)$ \\
Trigeminal function & $17(18)$ & $28(29)$ & $4(4)$ & $78(80)$ & $15(16)$ \\
Headache & $47(49)$ & $55(57)$ & $6(6)$ & $76(78)$ & $15(16)$
\end{tabular}


Table 6 SF36 results for four study populations

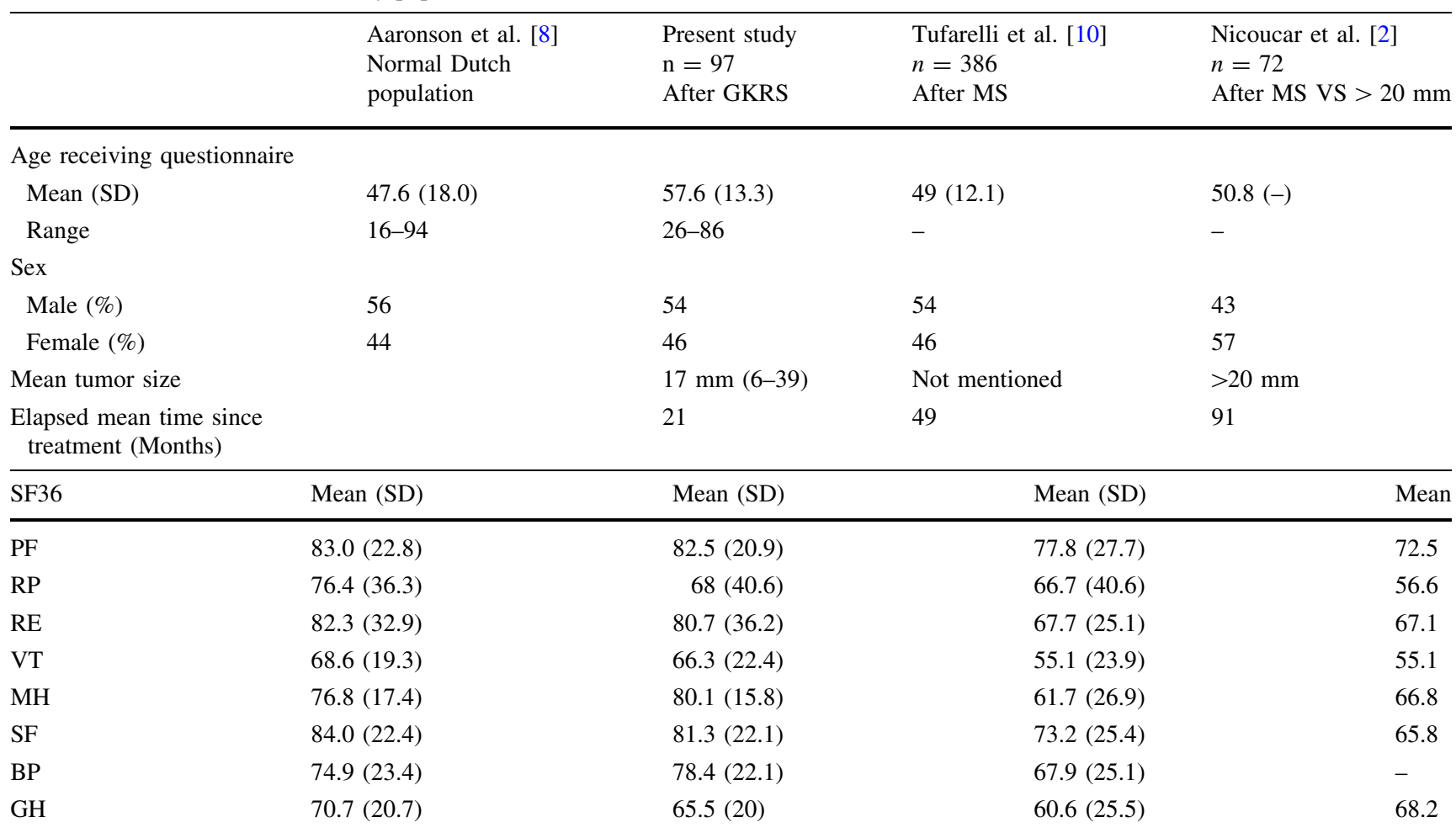

$P F$ physical functioning, $R P$ role limitations physical, $R E$ role limitations emotional, $V T$ vitality, $M H$ general mental health, $S F$ social functioning, $B P$ bodily pain, $G H$ general health, $\mathrm{PH}$ physical health, $E H$ emotional health, $S D$ standard deviation

\section{GBI}

The mean total GBI score we measured in our study group was -0.1 , which suggests a very small impact of GKRS on the QOL. However, the standard deviation (14.6) and the range (from -33.3 to 52.8 ) point at the presence of large inter-individual variations.

We have tried to identify individual variables that could explain these variations. In this search, we found some indication of a better QOL after treatment in patients who were diagnosed at an older age ( $>60$ years), but this outcome was not significant.
Like previous results reported by many authors including Myrseth et al., the three most common audiovestibular symptoms before and after GKRS were tinnitus, hearing loss, and unsteadiness [1]. The GBI results clearly show the influence of these symptoms on the QOL.

In the literature, we found one study that assessed QOL after MS by GBI [14]. To compare our results we divided our study population into three separate groups: patients whose QOL was better, worse or the same after GKRS (Table 7).

Nikolopoulos et al. [15] recorded a decreased QOL in $54 \%$ of his patients after MS compared to our $37 \%$ after
Table 7 QOL after GKRS or MS treatment, measured by GBI

\begin{tabular}{lll}
\hline & $\begin{array}{l}\text { Study population } \\
\text { After GKRS }(n=97)\end{array}$ & $\begin{array}{l}\text { Nikolopoulos et al. [13] } \\
\text { After MS }(n=53)\end{array}$ \\
\hline $\begin{array}{l}\text { Age receiving questionnaire } \\
\text { Mean (SD) }\end{array}$ & $57.6(13.3)$ & $49(-)$ \\
Range & $26-86$ & $25-76$ \\
Mean tumor size (mm) & 17 & 21.5 \\
Elapsed time since treatment (years) & Range 0.2-4.6 (mean 1.75) & Range 1-3 \\
QOL measured by GBI & & \\
Better (\%) & 39.4 & 17.4 \\
Same (\%) & 23.9 & 28.8 \\
Worse $(\%)$ & 36.7 & 53.8 \\
\hline
\end{tabular}


GKRS. The same applies to increased QOL, where they found $17 \%$ compared to our 39\%. Comparable results were found by Myrseth et al. They retrospectively compared their MS and GKRS results and concluded that posttreatment QOL, as well as facial nerve functions, hearing, and complication rate, were better after GKRS [4]. In their latest study, these results could be reconfirmed [13]. This retrospective study suggests that GKRS, when performed with a relatively low radiation dose, has a low impact on QOL in patients with VS.

To compare the QOL between patients treated by GKRS and MS a prospective study, preferably randomized, with a patient group matched on tumor size and pretreatment symptoms is required. In the present study, the audiovestibular symptoms were assessed retrospectively and could therefore be biased. Moreover, these symptoms were not objectively assessed. In that light, the results of this study are not fully comparable to those found in the literature.

Despite these shortcomings, this data offer further insight into the patient's opinion and perspective. This is important since decision-making in VS cases increasingly tends to be determined by the personal preference of the patients involved.

\section{Conclusion}

This study showed that GKRS for VS has little impact on the general QOL. However, the range of individual QOL results is wide. Individual QOL is influenced by the audiovestibular symptoms. No predictive patient, tumor, or treatment factors for QOL outcome after GKRS can be identified.

Acknowledgments The authors thank Martijn G. H. van Oijen for providing statistical advice.

Conflict of interest statement The authors declare that they have no conflict of interest.

Open Access This article is distributed under the terms of the Creative Commons Attribution Noncommercial License which permits any noncommercial use, distribution, and reproduction in any medium, provided the original author(s) and source are credited.

\section{References}

1. Myrseth E, Pedersen PH, Moller P, Lund-Johansen M (2007) Treatment of vestibular schwannomas. Why, when and how? Acta Neurochir (Wien) 149(7):647-660

2. Nicoucar K, Momjian S, Vader JP, De TN (2006) Surgery for large vestibular schwannomas: how patients and surgeons perceive quality of life. J Neurosurg 105(2):205-212

3. Tschudi DC, Linder TE, Fisch U (2000) Conservative management of unilateral acoustic neuromas. Am J Otol 21(5):722-728

4. Myrseth E, Moller P, Pedersen PH, Vassbotn FS, Wentzel-Larsen T, Lund-Johansen M (2005) Vestibular schwannomas: clinical results and quality of life after microsurgery or gamma knife radiosurgery. Neurosurgery 56(5):927-935

5. Regis J, Pellet W, Delsanti C, Dufour H, Roche PH, Thomassin JM et al (2002) Functional outcome after gamma knife surgery or microsurgery for vestibular schwannomas. J Neurosurg 97(5):1091-1100

6. Flickinger JC, Kondziolka D, Lunsford LD (1996) Dose and diameter relationships for facial, trigeminal, and acoustic neuropathies following acoustic neuroma radiosurgery. Radiother Oncol 41(3):215-219

7. Ware JE Jr, Sherbourne CD (1992) The MOS 36-item short-form health survey (SF-36). I. Conceptual framework and item selection. Med Care 30(6):473-483

8. Aaronson NK, Muller M, Cohen PD, Essink-Bot ML, Fekkes M, Sanderman R et al (1998) Translation, validation, and norming of the Dutch language version of the SF-36 health survey in community and chronic disease populations. J Clin Epidemiol 51(11):1055-1068

9. Robinson K, Gatehouse S, Browning GG (1996) Measuring patient benefit from otorhinolaryngological surgery and therapy. Ann Otol Rhinol Laryngol 105(6):415-422

10. Kanzaki J, Tos M, Sanna M, Moffat DA, Monsell EM, Berliner KI (2003) New and modified reporting systems from the consensus meeting on systems for reporting results in vestibular schwannoma. Otol Neurotol 24(4):642-648

11. Slavenburg S, van Oijen MG, Spiegel BM (2008) Comparison of health-related quality of life between populations. Liver Int 28(2):285-286

12. Tufarelli D, Meli A, Alesii A, De AE, Badaracco C, Falcioni M et al (2006) Quality of life after acoustic neuroma surgery. Otol Neurotol 27(3):403-409

13. Myrseth E, Moller P, Pedersen PH, Lund-Johansen M (2009) Vestibular schwannoma: surgery or gamma knife radiosurgery? A prospective, nonrandomized study. Neurosurgery 64(4):654-661

14. Chopra R, Kondziolka D, Niranjan A, Lunsford LD, Flickinger JC (2007) Long-term follow-up of acoustic schwannoma radiosurgery with marginal tumor doses of 12 to $13 \mathrm{~Gy}$. Int J Radiat Oncol Biol Phys 68(3):845-851

15. Nikolopoulos TP, Johnson I, O’Donoghue GM (1998) Quality of life after acoustic neuroma surgery. Laryngoscope 108(9):13821385 